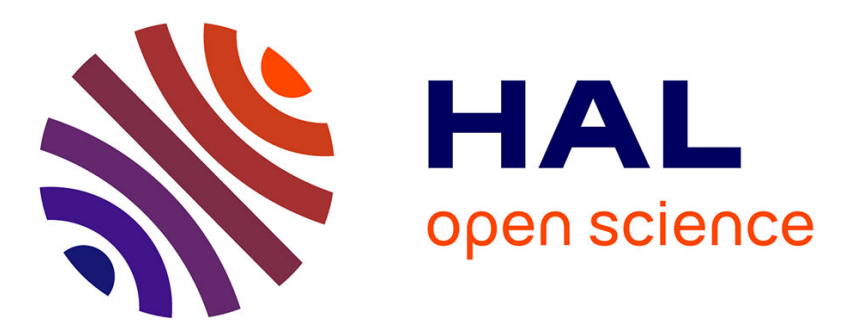

\title{
Conséquences de la mise en place des groupements hospitaliers de territoires sur les départements d'information médicale
}

\author{
Claire Le Pors, Carole Lê-Leplat, Isabelle Hirtzlin
}

\section{To cite this version:}

Claire Le Pors, Carole Lê-Leplat, Isabelle Hirtzlin. Conséquences de la mise en place des groupements hospitaliers de territoires sur les départements d'information médicale. Epidemiology and Public Health $=$ Revue d'Epidémiologie et de Santé Publique, 2018. hal-01731071

\section{HAL Id: hal-01731071 \\ https://hal.science/hal-01731071}

Submitted on 6 Apr 2018

HAL is a multi-disciplinary open access archive for the deposit and dissemination of scientific research documents, whether they are published or not. The documents may come from teaching and research institutions in France or abroad, or from public or private research centers.
L'archive ouverte pluridisciplinaire HAL, est destinée au dépôt et à la diffusion de documents scientifiques de niveau recherche, publiés ou non, émanant des établissements d'enseignement et de recherche français ou étrangers, des laboratoires publics ou privés. 
Conséquences de la mise en place des groupements hospitaliers de territoire sur les départements d'information médicale $\overbrace{\text { UNIVERSITÉ PARIS } 1}^{\text {A }}$

PANTHÉON SORBONNE

C. Le Pors ${ }^{1}$, C. Lê-Leplat ${ }^{1}$, I. Hirtzlin ${ }^{2}$

${ }^{1}$ Agence technique de linformation sur l'hospitalisation, Lyon, France

2 Université Paris 1, Centre d'économie de la Sorbonne

Introduction

La Loi de modernisation de notre système de santé du 26 janvier 2016 comporte un volet de mise en place de 135 groupements hospitaliers de territoire (GHT). L'information médicale fait partie des fonctions mutualisées et placées sous la responsabilité de l'établissement support. L'étude réalisée vise à présenter l'impact des GHT sur l'organisation et l'activité des départements d'information médicale (DIM).

\section{Méthode}

Dans un premier temps, une typologie des GHT a permis de dégager quatre catégories : les GHT comprenant un $\mathrm{CHU}$, les GHT sans $\mathrm{CHU}$ et constitués de 1 à 5 établissements, les GHT sans $\mathrm{CHU}$ et constitués de 6 à 14 établissements et les GHT composés uniquement d'EPSM (Etablissement public de santé mentale). On a pour cela exploité sous SAS des données issues de la base nationale PMSI 2015 et le référentiel des GHT fourni par la DGOS.

Une enquête qualitative a ensuite été réalisée afin de décrire l'état d'avancement de la mise en place des DIM de GHT et d'en identifier les déterminants et les impacts. Quatre DIM de GHT ont été choisis (dont deux avec $\mathrm{CHU}$ ) et 16 entretiens semi-directifs y ont été réalisés au printemps 2017. Les participants aux entretiens sont des membres du personnel du DIM de territoire (médecins DIM, TIM) et des membres de la direction des établissements.

\section{Résultats}

Analyse quantitative (base : référentiel DGOS GHT mai 2017)

Répartition des types de GHT dans

l'ensemble des GHT

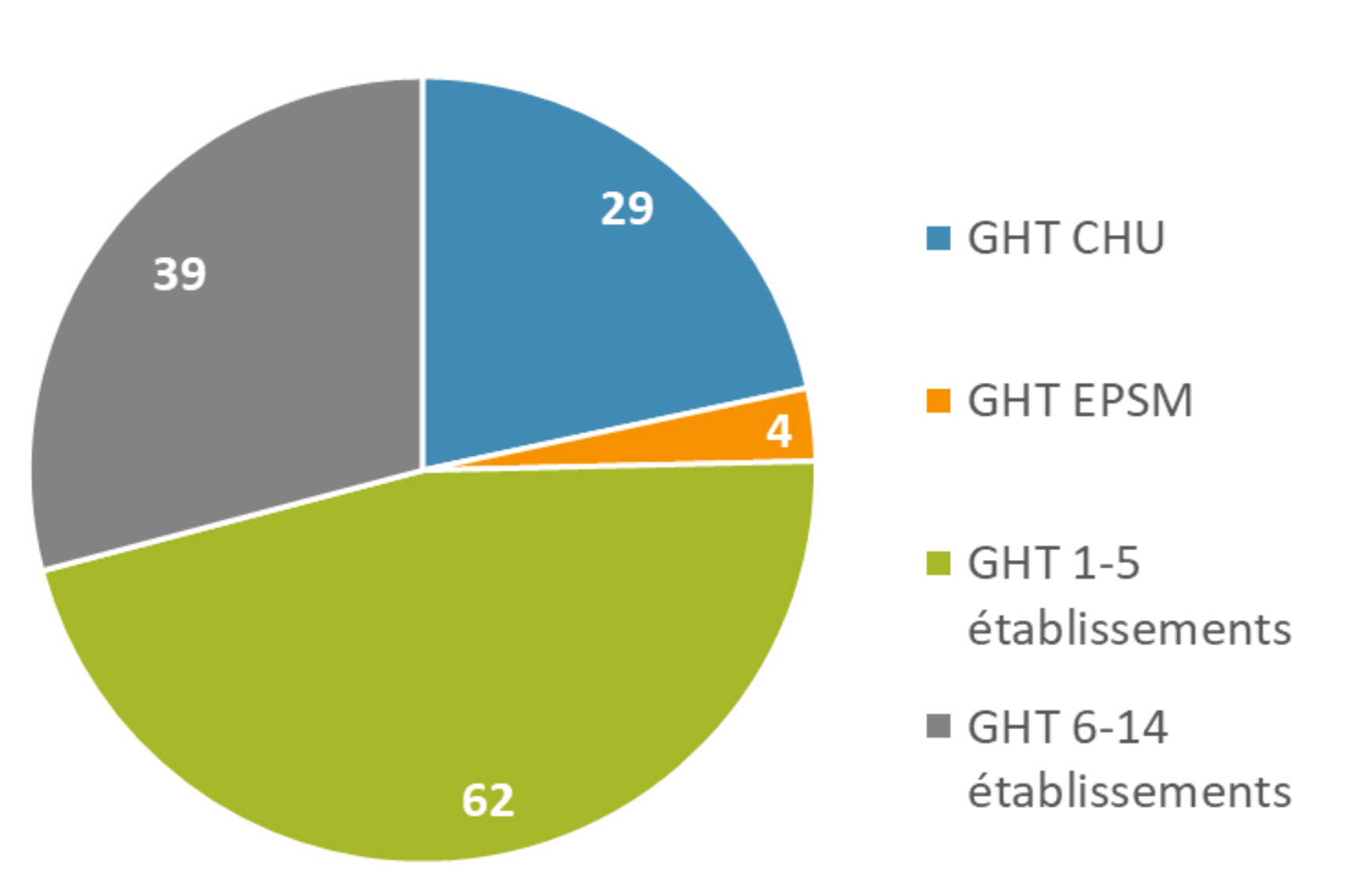

4 catégories:

- Les GHT comprenant un $\mathrm{CHU}$ Les GHT comprenant uniquemen des Établissements publics de santé mentale (EPSM) Les $\mathrm{GHT}$ sans $\mathrm{CHU}$ et qui regroupent 1 à 5 etablissements de santé 6 à 14 établissements de santé établissements établissements
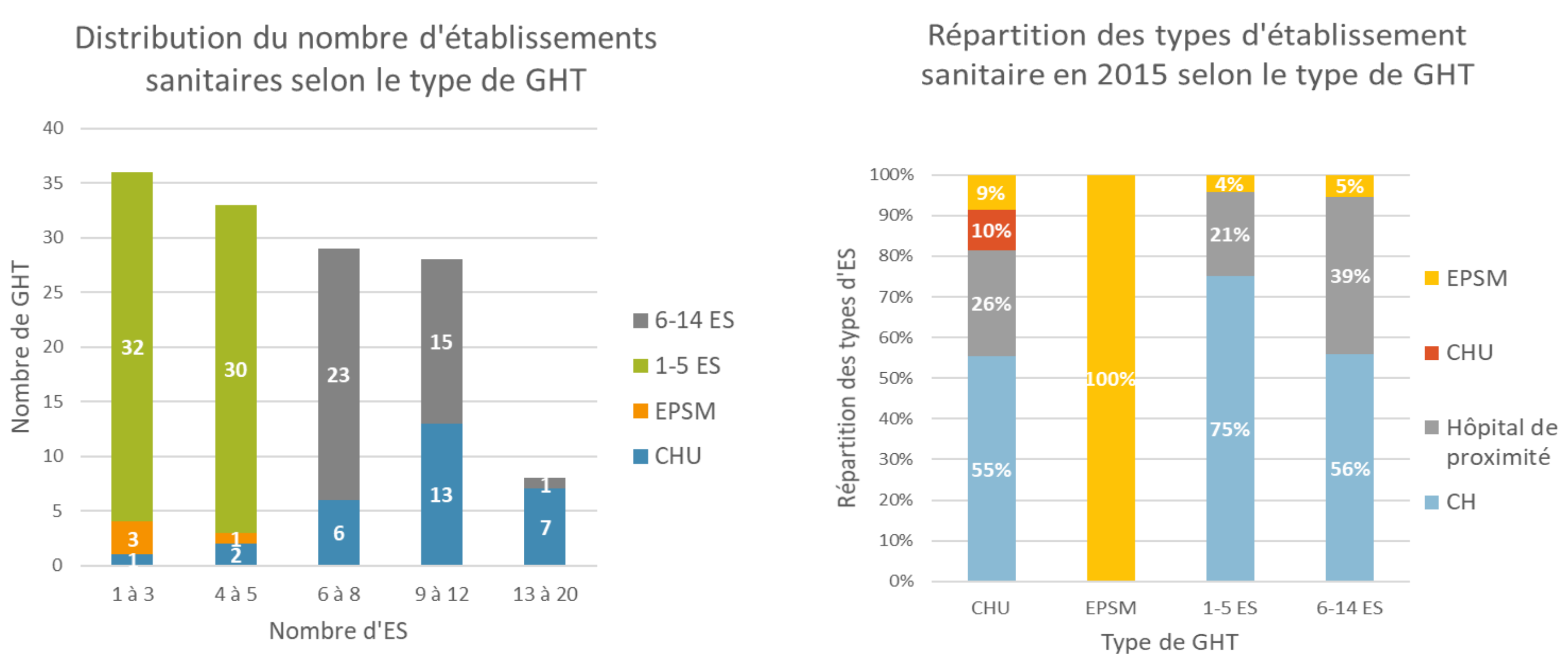

Analyse qualitative

Les données recueillies ont permis de réaliser quatre monographies de DIM de territoire.

\section{Etat d'avancement}

De manière générale, on a constaté que les principales avancées notables, au moment de l'enquête, étaient de deux types : la mise en place d'une organisation du DIM, et l'acquisition de premières expériences de travail en commun.
La participation au projet médical partagé a donné aux DIM une première occasion de collaborer au sein du DIM de territoire. Toutefois, les GHT avec $\mathrm{CHU}$ ont d'avantage sollicité le DIM que les autres.

Au-delà de cette première expérience commune, les DIM ont commencé à mettre en place des éléments d'organisation. Le MRIM (Médecin responsable de l'information médicale) de territoire a été désigné dans trois des quatre GHT. Deux d'entre eux ont également créé un bureau des DIM, composé d'au moins un représentant de chaque DIM d'établissement, et permettant une prise de décision plus collégiale. On retrouve également dans deux GHT des représentants filière, chargés d'assurer le lien entre le DIM et chaque filière de soins. Enfin, deux d'entre eux ont également mis en place des médecins référents pour chaque établissement.

Outre ces premiers travaux, de nombreux projets d'harmonisation ou de mutualisation des fonctions ont été évoqués. Toutefois, les DIM de territoire n'ayant été mis en place que récemment, peu de ces projets ont déjà été réalisés.

\section{Freins au développement des DIM de territoire}

Avant la création des GHT, le personnel des DIM avait déjà une charge de travail très importante. Or, toutes les missions relatives à leur établissement sont maintenues, mais s'y ajoutent celles liées au DIM de territoire. Cet accroissement de la charge de travail n'est pas compensé par un renforcement des moyens humains.

D'autre part, une base de données commune est nécessaire pour réaliser des analyses de l'activité à l'échelle du GHT. Or, au moment de l'enquête, aucun des DIM de territoire étudiés ne disposait de cette base de données. Beaucoup utilisaient donc les bases de données nationales de I'ATIH, qui sont anonymisées (par ex, identification précise des unités médicales impossible) mais qui permettent le chaînage et donc l'analyse du parcours du patient.

Enfin, les DIM de territoire manquent également de systèmes d'information communs ou convergents. Cet outil est très important, car il permet d'envisager une production et un envoi communs des données, ou encore des travaux d'identito-vigilance.

\section{$\underline{\text { Impact sur les conditions de travail }}$}

Les DIM ont évoqué plusieurs conséquences à la mutualisation des DIM de territoire, constatées ou attendues.

\begin{tabular}{|c|c|}
\hline Conséquences positives & Conséquences négatives \\
\hline $\begin{array}{l}\text { Moins d'isolement pour le personnel : la mise en } \\
\text { place des GHT a accru les échanges entre les } \\
\text { DIM, ce qui réduit l'isolement des DIM des plus } \\
\text { petits établissements, qui travaillent souvent } \\
\text { seuls. } \\
\text { - Exploitation de la complémentarité des DIM : la } \\
\text { structuration en DIM de GHT permet de mettre } \\
\text { en commun les ressources et compétences } \\
\text { propres à chacun des DIM d'établissement. De } \\
\text { plus, c'est l'occasion pour le personnel de se } \\
\text { spécialiser (en devenant, par exemple, le } \\
\text { référent d'une filière de soin). } \\
\text { Revalorisation de la fonction DIM : la mise en } \\
\text { place des GHT a renforcé la visibilité des DIM } \\
\text { auprès des établissements, notamment du fait } \\
\text { des nouveaux besoins d'analyse de l'activité à } \\
\text { l'échelle du territoire. }\end{array}$ & $\begin{array}{l}\text { Augmentation de la charge de travail } \\
\text { - Position ambiguë au sein du GHT : les } \\
\text { DIM sont placés à la fois sous la } \\
\text { responsabilité de l'établissement support } \\
\text { et de leur propre établissement. Leurs } \\
\text { interlocuteurs sont plus nombreux, avec } \\
\text { des attentes parfois contradictoires. } \\
\text { - Perte d'autonomie vis-à-vis de } \\
\text { l'établissement support : le DIM de } \\
\text { territoire est sous l'autorité fonctionnelle } \\
\text { de l'établissement support. Les DIM des } \\
\text { établissements parties craignent donc de } \\
\text { perdre toute autonomie. }\end{array}$ \\
\hline
\end{tabular}

Discussion et conclusion

Les résultats de cette étude complètent ceux des enquêtes par questionnaire déjà réalisées par le SDIM (Syndicat des DIM) et le CNEH (Centre national de l'expertise hospitalière). Ils offrent une vision plus détaillée des DIM de territoire étudiés. Cependant, ils ne peuvent être généralisés à l'ensemble des DIM de GHT. Le périmètre de cette étude gagnerait donc à être élargi dans l'espace (interroger d'autres DIM de GHT), ou dans le temps (reconduite de l'enquête à deux puis trois ans après la mise en place). 